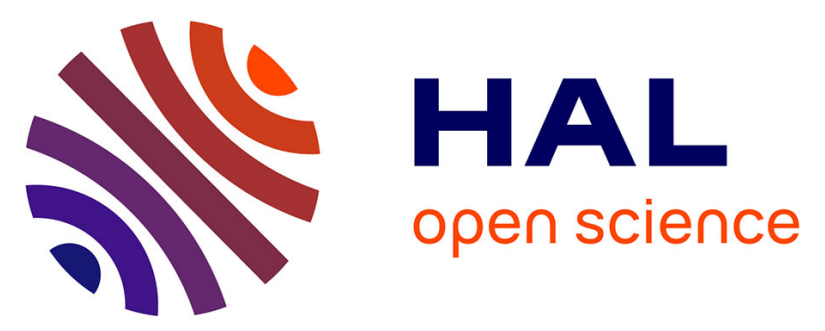

\title{
Validation study on urinary biomarkers of exposure for aflatoxin B-1, ochratoxin A, fumonisin B-1, deoxynivalenol and zearalenone in piglets
}

L. Gambacorta, M. Solfrizzo, A. Visconti, S. Powers, Anne Marie Cossalter,

Philippe Pinton, Isabelle P. Oswald

\section{To cite this version:}

L. Gambacorta, M. Solfrizzo, A. Visconti, S. Powers, Anne Marie Cossalter, et al.. Validation study on urinary biomarkers of exposure for aflatoxin B-1, ochratoxin A, fumonisin B-1, deoxynivalenol and zearalenone in piglets. World Mycotoxin Journal, 2013, 6 (3), pp.299 - 308. 10.3920/WMJ2013.1549 . hal-02650761

\section{HAL Id: hal-02650761 \\ https://hal.inrae.fr/hal-02650761}

Submitted on 7 Sep 2021

HAL is a multi-disciplinary open access archive for the deposit and dissemination of scientific research documents, whether they are published or not. The documents may come from teaching and research institutions in France or abroad, or from public or private research centers.
L'archive ouverte pluridisciplinaire HAL, est destinée au dépôt et à la diffusion de documents scientifiques de niveau recherche, publiés ou non, émanant des établissements d'enseignement et de recherche français ou étrangers, des laboratoires publics ou privés. 


\title{
Validation study on urinary biomarkers of exposure for aflatoxin $B_{1}$, ochratoxin $A$, fumonisin $B_{1}$, deoxynivalenol and zearalenone in piglets
}

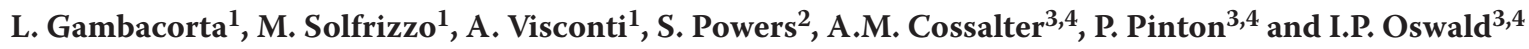 \\ ${ }^{1}$ Institute of Sciences of Food Production (ISPA), National Research Council of Italy (CNR), Via Amendola 122/o, 70126 \\ Bari, Italy; ${ }^{2}$ Vicam, 34 Maple Street, Milford, MA 01757, USA; ${ }^{3}$ INRA, UMR1331, Toxalim, Research Centre in Food \\ Toxicology, 180 Chemin de Tournefeuille, 31027 Toulouse, France; ${ }^{4}$ Université de Toulouse, INP, UMR1331, Toxalim, 180 \\ Chemin de Tournefeuille, 31000 Toulouse, France; michele.solfrizzo@ispa.cnr.it
}

Received: 23 January 2013 / Accepted: 14 May 2013

(c) 2013 Wageningen Academic Publishers

\section{RESEARCH PAPER}

\begin{abstract}
The multi-biomarker approach was used to validate urinary biomarkers in piglets administered boluses contaminated with mixtures of deoxynivalenol (DON), aflatoxin $\mathrm{B}_{1}\left(\mathrm{AFB}_{1}\right)$, fumonisin $\mathrm{B}_{1}\left(\mathrm{FB}_{1}\right)$, zearalenone $(\mathrm{ZEA})$ and ochratoxin A (OTA) at different concentrations. Boluses contaminated with mycotoxins were prepared by slurrying and freezedrying feed material fortified with culture extracts of selected toxigenic fungi. Piglets were individually placed in metabolic cages to collect urine before gavage and $24 \mathrm{~h}$ post dose. Urine samples were hydrolysed with $\beta$-glucuronidase and analysed by a multi-biomarker LC-MS/MS method developed and validated to identify and measure biomarkers of $\mathrm{FB}_{1}$, OTA, DON, ZEA and $\mathrm{AFB}_{1}$. Urinary levels of $\mathrm{FB}_{1}$, OTA, DON + de-epoxy-deoxynivalenol, ZEA + alphazearalenol and aflatoxin $\mathrm{M}_{1}$ were selected as biomarkers of $\mathrm{FB}_{1}$, OTA, DON, ZEA and $\mathrm{AFB}_{1}$, respectively. Mean percentages of dietary mycotoxins excreted as biomarkers in $24 \mathrm{~h}$ post dose urine were $36.8 \%$ for ZEA, $28.5 \%$ for DON, $2.6 \% \mathrm{FB}_{1}, 2.6 \%$ for $\mathrm{OTA}$ and $2.5 \%$ for $\mathrm{AFB}_{1}$. A good correlation was observed between the amount of mycotoxins ingested and the amount of relevant biomarkers excreted in $24 \mathrm{~h}$ post dose urine. Linear dose-response correlation coefficients ranged between 0.68 and 0.78 for the tested couples of mycotoxin/biomarker. The good sensitivity of the LC-MS/MS method and the good dose-response correlations observed in this study permitted to validate the selected mycotoxin biomarkers in piglets at dietary levels close to the maximum permitted levels reported in Commission Directive 2003/100/EC for $\mathrm{AFB}_{1}$ and the guidance values reported in Commission Recommendation 2006/576/EC for DON, ZEA, OTA and $\mathrm{FB}_{1}$.
\end{abstract}

Keywords: bioavailability, urine, excretion, dose-response

\section{Introduction}

Deoxynivalenol (DON), aflatoxin $\mathrm{B}_{1}\left(\mathrm{AFB}_{1}\right)$, fumonisin $\mathrm{B}_{1}\left(\mathrm{FB}_{1}\right)$, zearalenone $(\mathrm{ZEA})$ and ochratoxin $\mathrm{A}(\mathrm{OTA})$ are mycotoxins frequently occurring in cereals and cerealbased feeds. Farm animals are often exposed to these mycotoxins alone or in combinations (Grenier and Oswald, 2011; Streit et al., 2012). Levels of these contaminants in cereals and cereal-based feeds should be kept as low as possible to protect not only animal health but also human health, since some mycotoxins or their toxic metabolites can be released in animal products such as milk, meat and offal. In the European Union, maximum permitted levels for $\mathrm{AFB}_{1}$ in feed materials range from 0.005 to 0.02 $\mathrm{mg} / \mathrm{kg}$. Guidance values are reported in Commission Recommendation 2006/576/EC (EC, 2006) for DON (0.9-12 $\mathrm{mg} / \mathrm{kg})$, ZEA $(0.1-3 \mathrm{mg} / \mathrm{kg})$, OTA $(0.05-0.25 \mathrm{mg} / \mathrm{kg})$ and $\mathrm{FB}_{1}+$ fumonisin $\mathrm{B}_{2}(5-60 \mathrm{mg} / \mathrm{kg})$. An indirect approach to reduce the negative impact of mycotoxins on animal health is the use of mycotoxin detoxifying-agents leading to a decreased bioavailability of mycotoxins (Boudergue et al., 2009). Several types of mycotoxin-detoxifying agents are commercially available and largely used by farmers (Avantaggiato et al., 2005). Some of them have been shown to efficiently work in vitro, but only a few have been tested in vivo (Boudergue et al., 2009; Meissonnier et al. 2009; 
Solfrizzo et al., 2001a,b). Moreover, data on the efficacy of commercial binders to adsorb mixtures of mycotoxins are very scarce or absent. In recent guidelines, the European Food Safety Authority (EFSA) has stated that in vitro tests do not fully prove the efficacy of mycotoxin-detoxifying agents and that in vivo trials should be performed (EFSA, 2010). Moreover, in vivo trials have to be performed at mycotoxin levels below those reported in Directive 2003/100/EC for $\mathrm{AFB}_{1}$ (EC, 2003) and Recommendation 2006/576/EC for DON, ZEA, OTA and $\mathrm{FB}_{1}$ (EC, 2006).

The measurement of urinary mycotoxin biomarkers is a promising approach to assess the in vivo efficacy of binders, since a portion of ingested mycotoxin is released into urine as parent toxin or phase I and II metabolites. Therefore, their measurement could give an estimate of the ingested dose. Recently, EFSA has confirmed the validity of the biomarker approach for demonstration of the efficacy of substances that reduce the mycotoxin contamination of feed (EFSA, 2010). Mycotoxin biomarkers are present in biological fluids and urine is becoming the choice for the measurement of mycotoxin biomarkers (De Andrés et al., 2008; Polychronaki et al., 2008; Scott, 2005; Shephard et al., 2007; Turner et al., 2010). Urinary biomarkers for $\mathrm{FB}_{1}$, OTA, DON, ZEA and $\mathrm{AFB}_{1}$ could be $\mathrm{FB}_{1}$, OTA, DON + de-epoxydeoxynivalenol (DOM-1), ZEA+alpha-zearalenol ( $\alpha-Z \mathrm{ZO})$ + beta-zearalenol $\left(\beta\right.$-ZOL) and aflatoxin $\mathrm{M}_{1}\left(\mathrm{AFM}_{1}\right)$, respectively. Recent developments in the field of analytical chemistry of mycotoxins have demonstrated the power of liquid chromatography tandem mass spectrometry (LCMS/MS) in the simultaneous determination of mycotoxin biomarkers in urine (Ediage et al., 2012; Solfrizzo et al., 2011; Warth et al., 2012). There is a need of validated multimycotoxin biomarkers that can be used in animal studies to evaluate the efficacy of mycotoxin detoxifying agents capable to reduce the bioavailability of mycotoxin mixtures.

The objective of this study was to evaluate the doseresponse relationship between the simultaneous oral ingestion of $\mathrm{FB}_{1}$, OTA, DON, ZEA and $\mathrm{AFB}_{1}$ and urinary excretion of the relevant biomarkers within $24 \mathrm{~h}$ by using a mass balance approach.

\section{Material and methods}

\section{Chemicals and reagents}

Calibrant solutions of DON $(100 \mu \mathrm{g} / \mathrm{ml})$, DOM-1 $(50 \mu \mathrm{g} / \mathrm{ml}), \mathrm{AFB}_{1}+$ aflatoxin $\mathrm{B}_{2}+$ aflatoxin $\mathrm{G}_{1}+$ aflatoxin $\mathrm{G}_{2}(2.02 \mu \mathrm{g} / \mathrm{ml}, 0.5 \mu \mathrm{g} / \mathrm{ml}, 2.03 \mu \mathrm{g} / \mathrm{ml}$ and $0.5 \mu \mathrm{g} / \mathrm{ml}$, respectively), $\operatorname{AFM}_{1}(0.5 \mu \mathrm{g} / \mathrm{ml}), \alpha-Z O L(10 \mu \mathrm{g} / \mathrm{ml}), \beta-Z O L$ $(10 \mu \mathrm{g} / \mathrm{ml})$, ZEA $(100 \mu \mathrm{g} / \mathrm{ml})$, OTA $(10 \mu \mathrm{g} / \mathrm{ml})$ prepared in acetonitrile $(\mathrm{ACN})$ and a calibrant solution of $\mathrm{FB}_{1}(50 \mu \mathrm{g} / \mathrm{ml})$ prepared in ACN:water (50:50, v/v) were purchased from Romer Labs Diagnostic (Tulln, Austria). $\beta$-glucuronidase/ sulfatase type $\mathrm{H}-2$ from Helix pomatia (specific activity
130,200 units/ml $\beta$-glucuronidase, 709 units/ml sulfatase), chromatography-grade methanol $(\mathrm{MeOH})$, acetonitrile, acetone, glacial acetic acid and phosphate buffered saline (PBS) tablets were purchased from Sigma-Aldrich (Milan, Italy). PBS solution at $\mathrm{pH} 7.4$ was prepared by dissolving PBS tablets in an appropriate volume of distilled water. Sodium hydrogen carbonate was purchased from J.T. Backer (Deventer, the Netherlands). Ultrapure water was produced by use of the Milli-Q system (Millipore, Bedford, MA, USA). Myco6in1, DONtest ${ }^{\mathrm{mm}}$ Wide Bore (WB), AflaTest ${ }^{\mathrm{m}}$ WB, FumoniTest ${ }^{\mathrm{mw}} \mathrm{WB}$, ZearaleTest ${ }^{\mathrm{mw}} \mathrm{WB}$ and OchraTest $^{\mathrm{mw}}$ WB immunoaffinity columns were purchased from Vicam L.P. (Watertown, MA, USA). Oasis HLB cartridges (60 mg, $3 \mathrm{ml}$ ) were purchased from Waters (Milford, MA, USA) and regenerated cellulose filters $(0.45 \mu \mathrm{m})$ were purchased from Sartorius Stedim Biotech (Goettingen, Germany). No. 4 and GF/A paper and glass microfiber filters were obtained from Whatman (Maidstone, UK).

\section{Design of the in vivo experiments with piglets}

In vivo experiments with piglets were performed to check the feasibility of using urinary multi-biomarker determination as a suitable approach to estimate the ingestion of mixtures of $\mathrm{DON}, \mathrm{AFB}_{1}, \mathrm{FB}_{1}$, ZEA and OTA at different levels in feed boluses. Sixteen 4-week-old weaned piglets (Pietrain/Duroc/Large-white) weighing $10.56 \pm 1.88 \mathrm{~kg}$ at the beginning of the experiment were obtained locally. Animals were acclimatised for 1 week in the animal facility of the INRA ToxAlim Unit (Toulouse, France) prior to being used in experimental protocols. During the acclimation and experimental periods, animals were given free access to water. Except during the urine collection periods, animals were fed a commercial diet ad libitum. Four groups of piglets (four piglets per group) were administered boluses contaminated with mixtures of DON, $\mathrm{AFB}_{1}, \mathrm{FB}_{1}, \mathrm{ZEA}$ and $\mathrm{OTA}$ at different levels (for mycotoxin intake, Table 1). Each piglet was fed the bolus and then housed in a metabolic cage. Urine samples were collected 3 times at regular intervals within $24 \mathrm{~h}$ and their volumes were measured. Control urine samples were collected from the same piglet the day before giving the contaminated bolus. Urine samples were frozen and shipped to ISPA (Bari, Italy) for determination of DON, DOM-1, $\mathrm{AFM}_{1}$, ZEA, $\alpha$-ZOL, $\beta$-ZOL, $\mathrm{FB}_{1}$ and OTA.

\section{Preparation of contaminated feed boluses}

About $2 \mathrm{~kg}$ of commercial complete feed for piglets was homogenized, analysed for mycotoxins and used for preparation of mycotoxin-contaminated boluses as well as for recovery experiments of $\mathrm{DON}, \mathrm{AFB}_{1}, \mathrm{FB}_{1}, \mathrm{ZEA}$ and OTA at ISPA. For the preparation of contaminated boluses, an aliquot of feed was ground and fortified with culture extracts of mycotoxigenic species of Fusarium graminearum, Aspergillus ochraceus, Aspergillus parasiticus 
Table 1. Mycotoxin intakes in four groups of piglets that received a bolus containing a mixture of deoxynivalenol, aflatoxin $B_{1}$, fumonisin $B_{1}$, zearalenone and ochratoxin $A$.

\begin{tabular}{|c|c|c|c|c|c|c|c|c|}
\hline \multirow[t]{2}{*}{ Mycotoxin } & \multicolumn{2}{|c|}{ Bolus $1(n=4)^{1}$} & \multicolumn{2}{|c|}{ Bolus $2(n=4)^{2}$} & \multicolumn{2}{|c|}{ Bolus $3(n=4)^{3}$} & \multicolumn{2}{|c|}{ Bolus $4(n=4)^{4}$} \\
\hline & $\mu \mathrm{g} / \mathrm{kg}$ bw & $\mu \mathrm{g} /$ animal & $\mu \mathrm{g} / \mathrm{kg}$ bw & $\mu \mathrm{g} / \mathrm{animal}$ & $\mu \mathrm{g} / \mathrm{kg}$ bw & $\mu \mathrm{g} /$ animal & $\mu \mathrm{g} / \mathrm{kg}$ bw & $\mu g /$ animal \\
\hline Deoxynivalenol & 7.16 & 63.61 & 20.44 & 191.12 & 24.14 & 315.05 & 57.38 & 630.03 \\
\hline Aflatoxin $B_{1}$ & 0.16 & 1.40 & 0.45 & 4.20 & 0.54 & 7.03 & 1.28 & 14.01 \\
\hline Fumonisin $\mathrm{B}_{1}$ & 3.71 & 32.96 & 10.60 & 99.14 & 63.19 & 824.60 & 150.19 & $1,649.12$ \\
\hline Zearalenone & 0.68 & 6.05 & 1.94 & 18.15 & 2.38 & 31.08 & 5.66 & 62.15 \\
\hline Ochratoxin A & 0.16 & 1.45 & 0.46 & 4.35 & 0.56 & 7.25 & 1.32 & 14.50 \\
\hline
\end{tabular}

and Fusarium verticillioides (deposited at ISPA Collection, http://www.ispa.cnr.it/Collection) producing DON and ZEA, OTA, aflatoxins and fumonisins, respectively, cultured on cereals. Each fungal culture was dried, ground and separately extracted with water or mixtures of water and organic solvent. In particular, fumonisins were extracted with water, OTA with a mixture of water and ACN (40:60, $\mathrm{v} / \mathrm{v}$ ) and aflatoxins with a mixture of water and acetone $(15: 85, \mathrm{v} / \mathrm{v})$. DON + ZEA were extracted from two portions of cereal culture with water and with a mixture of water and ACN (10:90, v/v) to obtain two different cultural extracts containing the necessary concentrations of DON + ZEA. The determination of each mycotoxin in these culture extracts was performed by HPLC-UV/FLD as described below. The concentration of 3-acetyl-DON (3-ADON) in extracts of $F$. graminearum was also measured by HPLC$\mathrm{UV}$, however, as the amount of 3-ADON measured in these extracts was $<2 \%$ than that of DON, it was not considered for the mass balance. For the preparation of boluses contaminated with four different mixtures of DON, $\mathrm{AFB}_{1}, \mathrm{FB}_{1}, \mathrm{ZEA}$ and OTA, four aliquots of ground feed were contaminated with appropriate volumes of culture extracts necessary to reach the level of each mycotoxin as reported in Table 1 . These four aliquots were separately slurried to ensure within sample homogenisation, then divided into $4 \times 4$ boluses that were separately freeze-dried and delivered to INRA for in vivo experiment with piglets. These in vivo experiments were performed during two different periods. The first experiment was performed with 2 groups of 4 piglets that were administered the two boluses (50 g) containing higher mycotoxin concentrations (bolus 3 and bolus 4; Table 1). The second experiment was performed with the other 2 groups of 4 piglets that were administered the two boluses ( $20 \mathrm{~g}$ ) containing lower mycotoxin concentrations (bolus 1 and bolus 2; Table 1). A reduced weight of the bolus was used in the second experiment in order to decrease the time necessary to administer the bolus to the piglet. The feed was removed the evening before the experiment, thus the animal had no access to feed overnight. The animal keeper stayed with the animals while they were eating the bolus and verified that all the bolus was taken up by the animal. Depending on the animal, the bolus was eaten within 1 to $2 \mathrm{~h}$.

\section{Mycotoxin analysis in feed and culture extracts}

The concentrations of DON, aflatoxins, ZEA, OTA and fumonisins in the feed used for the in vivo experiments were measured by analysing representative aliquots of ground feed with the relevant HPLC-based standard methods. In particular, DON, aflatoxins, ZEA, OTA and fumonisins were analysed by using EN 15791:2009, EN/ISO 17375:2006, EN 15792:2009, EN 16007:2012 and EN 16006:2012, respectively (CEN, 2006, 2009a,b, 2012a,b). Method performances were checked with recovery experiments of duplicate feed samples spiked at the following levels: $0.162 \mu \mathrm{g} / \mathrm{g}$ DON, $0.0136 \mu \mathrm{g} / \mathrm{g} \mathrm{AFB}_{1}, 4.416 \mu \mathrm{g} / \mathrm{g} \mathrm{FB}_{1}, 0.058$ $\mu \mathrm{g} / \mathrm{g}$ ZEA and $0.0044 \mu \mathrm{g} / \mathrm{g}$ OTA. Spiked samples were left overnight at room temperature to allow solvent evaporation and equilibration between analytes and matrix. Percentage recovery and repeatability of results were acceptable for all tested mycotoxins, i.e. $105 \pm 3.6 \% \mathrm{DON}, 102 \pm 1.7 \% \mathrm{AFB}_{1}$, $88 \pm 1.9 \% \mathrm{FB}_{1}, 95 \pm 0.5 \% \mathrm{ZEA}$ and $95 \pm 2 \%$ OTA. Analysis of the feed used for in vivo experiments showed the absence of $\mathrm{AFB}_{1}$ and $\mathrm{FB}_{1}$ and low levels of DON (104 $\left.\mu \mathrm{g} / \mathrm{kg}\right), \mathrm{ZEA}$ $(8 \mu \mathrm{g} / \mathrm{kg})$ and OTA $(0.17 \mu \mathrm{g} / \mathrm{kg})$. These low levels were considered acceptable as blank control samples. Therefore, an aliquot of this feed was used as a blank control diet whereas other aliquots were spiked with culture extracts to produce boluses contaminated with different concentrations of each mycotoxin.

The determination of DON, ZEA, OTA or $\mathrm{AFB}_{1}$ in each culture extract was performed by direct injection of culture 
extracts into HPLC-UV/FLD after appropriate dilution with water and filtration. For the determination of $\mathrm{FB}_{1}$ in the water culture extract of F. verticillioides, it was necessary to purify an aliquot of culture extract through an immunoaffinity column before HPLC-FLD analysis. The sample clean-up protocol and HPLC conditions were the same as those described above for the analysis of feed (EN 16006:2012; CEN, 2012a). Direct analysis of diluted culture extract was not possible due to the presence of an interfering peak co-eluting with $\mathrm{FB}_{1}$.

\section{Biomarker analysis in urine}

Three urine samples of each piglet collected during $24 \mathrm{~h}$ were pooled and analysed according to the multi-biomarker method based on multi-antibody immunoaffinity column (Myco6in1) and LC-MS/MS determination as described elsewhere (Solfrizzo et al., 2011) with some modifications. In particular, ZEA was added in the panel of biomarkers. Briefly, centrifuged urine samples $(6 \mathrm{ml})$ were incubated with $300 \mu$ l of $\beta$-glucuronidase/sulphatase solution $\left(37^{\circ} \mathrm{C}\right.$ for $18 \mathrm{~h}$ ), diluted with $6 \mathrm{ml}$ water and cleaned up on a Myco6in1 immunoaffinity column (IAC) (Vicam) and an OASIS HLB solid phase extraction (SPE) column (Waters) connected in tandem. After sample application, the two columns were separated; the IAC column was washed with $4 \mathrm{ml}$ water and biomarkers were eluted with $3 \mathrm{ml}$ methanol and $2 \mathrm{ml}$ water. The OASIS column was washed with $1 \mathrm{ml}$ methanol:water (20:80, v/v) and DON and DOM-1 passed through the IAC and collected on the SPE column were eluted with $1 \mathrm{ml}$ methanol:water (40:60, v/v). The separate eluates from the two columns were combined, dried down and reconstituted in $200 \mu \mathrm{l}$ methanol:water $(20: 80, \mathrm{v} / \mathrm{v})$ with $0.5 \%$ acetic acid. 5 -points matrix-assisted calibration curves were prepared in the following concentration range for each biomarker: 2-1,254 ng/ml DON, 1-250 ng/ml DOM-1, $0.2-25 \mathrm{ng} / \mathrm{ml} \mathrm{AFM}_{1}$, 2-315 ng/ml ZEA, 2-250 ng/ml $\alpha$ - and $\beta$-ZOL, 0.4-157 ng/ml FB 1 and 0.02-13 ng/ml OTA. In particular, aliquots of control urine samples were pooled and sub-aliquots were used to prepare the purified extracts for the 5-points matrix-assisted calibration curves. Table 2 shows the results of matrix effect determination (\% signal suppression enhancement (SSE)) for each biomarker in piglet urine, which are compared to results previously obtained for human urine. The \%SSE values were calculated as the ratio between the slope of the matrix-matched calibration curve and the slope of the calibration curve in LC mobile phase multiplied by 100 . Signal suppression was observed for all biomarkers in piglets and human urine except for $\mathrm{FB}_{1}$, for which signal enhancement was observed. Piglet urine showed a stronger signal suppression of DON, DOM-1, AFM $1, \alpha-Z O L, Z E A$ and OTA than human urine. The signal suppression of $\beta-Z O L$ as well as the signal enhancement of $\mathrm{FB}_{1}$ were lower in piglets.

\section{Equipment}

DON, $\mathrm{AFB}_{1}$, ZEA and OTA determinations in feed and culture extracts were performed with an Agilent 1100 series HPLC apparatus equipped with a G1312A binary pump, G1313 Autosampler, G1316A column thermostat set at $30{ }^{\circ} \mathrm{C}, \mathrm{G} 1315 \mathrm{~B}$ UV-visible DAD, G1321A spectrofluorometric detector, Agilent Chemstation G2170AA Windows 2000 operating system (Agilent, Waldbronn, Germany) and a post-column photochemical derivatizator (UVE Derivatizer, LC Tech, Dorfen, Germany). $\mathrm{FB}_{1}$ and $\mathrm{FB}_{2}$ determination was performed with a Varian HPLC system equipped with a ternary pump (ProStar 230),

Table 2. Comparison results from determination of matrix effects for each biomarker between piglet and human urine.

\begin{tabular}{|c|c|c|c|c|c|c|c|c|}
\hline \multirow[t]{2}{*}{ Biomarker $^{1}$} & \multicolumn{3}{|c|}{$\begin{array}{l}\text { Matrix-matched calibration curve in } \\
\text { piglet urine }{ }^{2}\end{array}$} & \multicolumn{3}{|c|}{$\begin{array}{l}\text { Matrix-matched calibration curve in } \\
\text { human urine } e^{2}\end{array}$} & \multirow{2}{*}{$\begin{array}{l}\text { Matrix effect } \\
\text { in piglet urine } \\
(\mathrm{SSE} \%)^{3}\end{array}$} & \multirow{2}{*}{$\begin{array}{l}\text { Matrix effect } \\
\text { in human } \\
\text { urine (SSE \%) }\end{array}$} \\
\hline & $a$ & b & $r^{2}$ & a & b & $r^{2}$ & & \\
\hline DON & 591 & 2,510 & 0.9990 & 1,942 & 2,565 & 0.9968 & 12 & 43 \\
\hline DOM-1 & 1,846 & 4,623 & 0.9996 & 3,147 & -4 & 0.9786 & 18 & 76 \\
\hline $\mathrm{AFM}_{1}$ & 740 & 84 & 0.9995 & 1,928 & -66 & 0.9966 & 33 & 81 \\
\hline $\mathrm{FB}_{1}$ & 144 & -74 & 0.9877 & 1,468 & -9 & 0.9938 & 152 & 270 \\
\hline a-ZOL & 114 & 375 & 0.9964 & 918 & -320 & 0.9939 & 14 & 28 \\
\hline$\beta-Z O L$ & 113 & 1,668 & 0.9884 & 292 & 122 & 0.9994 & 29 & 15 \\
\hline ZEA & 842 & -393 & 0.9902 & 3,753 & -881 & 0.9962 & 44 & 48 \\
\hline OTA & 2,940 & 241 & 0.9961 & 9,396 & 116 & 0.9905 & 42 & 77 \\
\hline
\end{tabular}


autosampler (ProStar 410), fluorescence detector (ProStar 363) and Varian Star Data System 6.20 (Varian Inc., Palo Alto, CA, USA).

LC-MS/MS of urinary biomarkers was performed with a QTrap MS/MS system (Applied Biosystems, Foster City, CA, USA), equipped with an ESI interface, an 1100 series micro-LC system comprising a binary pump, a microautosampler (Agilent Tecnhnologies) and Analyst 1.4 acquisition data system (Applied Biosystems).

Linear regression analyses were performed using SigmaPlot for Windows Version 12.0 statistical software package (Sistat Software, Inc., Chicago, IL, USA).

\section{Results and discussion}

The sensitivity of the LC-MS/MS method used in this study was adequate to detect and quantify the mycotoxin biomarkers excreted in urine of piglets administered boluses contaminated at reasonably low levels of $\mathrm{AFB}_{1}, \mathrm{OTA}, \mathrm{FB}_{1}$, DON and ZEA. The values of mycotoxin intake in the four groups of piglets used in this study are reported in Table 1. For DON and ZEA, the intake values were comparable with those used by Dänicke et al. (2005). For FB $\mathrm{FB}_{1}$, they were much lower than those used in previous studies with pigs (Dilkin et al., 2010; Fodor et al., 2008). For $\mathrm{AFB}_{1}$, they were lower than those used previously in studies with pigs (Dilkin et al., 2003; Ho, 1987; Thieu and Pettersson, 2009). For OTA, they were much lower than those used by Stoev et al. (2002) and comparable or slightly lower than those used by Stoev et al. (2001). The values of mycotoxin intake used in this study were chosen considering the maximum permitted levels and guidance levels reported in Directive 2003/100/EC (EC, 2003) and Recommendation 2006/576/ EC (EC, 2006), respectively. Considering that the piglets used in this study consumed about 350-400 g feed/day, the values of mycotoxin intake were below the limits for all tested doses of OTA and $\mathrm{FB}_{1}$. For $\mathrm{AFB}_{1}$, DON and ZEA, these values were below the limits for three out of the four doses and about twice the limits for the highest dose.

Table 3 shows the mean biomarker values (concentration and absolute amount of each biomarker excreted) found in the $24 \mathrm{~h}$ post dose urine samples collected from the four groups of piglets administered mixtures of five mycotoxins at four levels. All mycotoxin biomarkers were detected and measured in all urine samples with the exception of $\beta$-ZOL, which was detected and measured only in urines of piglets administered the highest dose of ZEA $(5.66 \mu \mathrm{g} / \mathrm{kg} \mathrm{bw})$. The presence of low concentrations of $\beta-Z O L$, compared to $\alpha-Z O L$, in urine of pigs fed with diet contaminated with ZEA was also reported by Dänicke et al. (2005). In particular, for urines containing both $\beta$-ZOL and $\alpha-Z O L$, the ratio $\alpha-Z O L / \beta-Z O L$ ranged between $17.5-73.7$, compared to 36.5 obtained in the present study. The mean percentage of mycotoxin ingested that was excreted in $24 \mathrm{~h}$ urine as biomarker(s) is shown in Figure 1 for $\mathrm{AFB}_{1}$, OTA, $\mathrm{FB}_{1}, \mathrm{DON}$ and $\mathrm{ZEA}$. The measurement of urine volume from each piglet and the mass balance of each analyte in the boluses and in the urine permitted to calculate the relevant percentage of ingested mycotoxin that was excreted as

Table 3. Urinary excretion of mycotoxin biomarkers. Piglets received a bolus containing a mycotoxin mixture ${ }^{1}$ and urinary excretion was measured during the next $24 \mathrm{~h}$. Results are reported as mean \pm standard deviation.

\begin{tabular}{|c|c|c|c|c|c|c|c|c|}
\hline \multirow[t]{2}{*}{ Biomarker ${ }^{2}$} & \multicolumn{2}{|l|}{ Bolus $1(n=4)^{3}$} & \multicolumn{2}{|l|}{ Bolus $2(n=4)^{4}$} & \multicolumn{2}{|l|}{ Bolus $3(n=4)^{5}$} & \multicolumn{2}{|l|}{ Bolus $4(n=4)^{6}$} \\
\hline & $\mathrm{ng} / \mathrm{ml}$ & $\mu g / a n i m a l$ & $\mathrm{ng} / \mathrm{ml}$ & $\mu g / a n i m a l$ & $\mathrm{ng} / \mathrm{ml}$ & $\mu g / a n i m a l$ & $\mathrm{ng} / \mathrm{ml}$ & $\mu \mathrm{g} /$ animal \\
\hline DON & $80.55 \pm 28.96$ & $23.35 \pm 12.96$ & $125.06 \pm 41.64$ & $34.57 \pm 15.09$ & $305.94 \pm 143.17$ & $128.65 \pm 59.04$ & $218.18 \pm 33.35$ & $101.32 \pm 49.97$ \\
\hline DOM-1 & $1.19 \pm 1.38$ & $0.25 \pm 0.29$ & $3.36 \pm 1.35$ & $0.90 \pm 0.40$ & $3.44 \pm 4.14$ & $1.42 \pm 1.64$ & $16.33 \pm 5.95$ & $7.65 \pm 5.24$ \\
\hline $\mathrm{AFM}_{1}$ & $0.14 \pm 0.12$ & $0.03 \pm 0.02$ & $0.36 \pm 0.31$ & $0.09 \pm 0.07$ & $0.50 \pm 0.31$ & $0.21 \pm 0.12$ & $0.88 \pm 0.35$ & $0.43 \pm 0.33$ \\
\hline $\mathrm{FB}_{1}$ & $1.55 \pm 0.21$ & $0.48 \pm 0.36$ & $4.82 \pm 2.72$ & $1.22 \pm 0.43$ & $77.37 \pm 43.62$ & $32.47 \pm 17.57$ & $117.78 \pm 71.91$ & $62.69 \pm 54.53$ \\
\hline ZEA & $11.63 \pm 7.52$ & $2.83 \pm 1.17$ & $23.06 \pm 11.42$ & $5.86 \pm 1.40$ & $15.76 \pm 9.62$ & $6.55 \pm 3.74$ & $17.08 \pm 4.15$ & $8.44 \pm 5.45$ \\
\hline a-ZOL & $3.60 \pm 3.70$ & $0.74 \pm 0.74$ & $6.76 \pm 4.43$ & $1.69 \pm 0.74$ & $5.24 \pm 3.29$ & $2.18 \pm 1.30$ & $6.58 \pm 2.36$ & $2.96 \pm 1.49$ \\
\hline$\beta-\mathrm{ZOL}^{7}$ & nd & nd & nd & nd & nd & nd & $0.39 \pm 0.36$ & $0.17 \pm 0.16$ \\
\hline OTA & $0.12 \pm 0.05$ & $0.04 \pm 0.02$ & $0.65 \pm 0.22$ & $0.17 \pm 0.02$ & $0.52 \pm 0.15$ & $0.22 \pm 0.06$ & $0.36 \pm 0.12$ & $0.15 \pm 0.01$ \\
\hline
\end{tabular}

\footnotetext{
${ }^{1}$ Mycotoxin mixture containing deoxynivalenol (DON), aflatoxin $\mathrm{B}_{1}$, fumonisin $\mathrm{B}_{1}\left(\mathrm{FB}_{1}\right)$, zearalenone (ZEA) and ochratoxin (OTA); see Table 1 for details.

${ }^{2}$ DOM-1 = de-epoxy-deoxynivalenol, $\mathrm{AFM}_{1}=$ aflatoxin $\mathrm{M}_{1}, \mathrm{a}-\mathrm{ZOL}=\mathrm{a}$-zearalenol, $\beta$-ZOL $=\beta$-zearalenol.

${ }^{3}$ Mean volume of $24 \mathrm{~h}$ urine $296.75 \pm 173.47 \mathrm{ml}$.

${ }^{4}$ Mean volume of $24 \mathrm{~h}$ urine $275.25 \pm 59.19 \mathrm{ml}$.

${ }^{5}$ Mean volume of $24 \mathrm{~h}$ urine $421.25 \pm 19.31 \mathrm{ml}$.

${ }^{6}$ Mean volume of $24 \mathrm{~h}$ urine $462.50 \pm 188.70 \mathrm{ml}$.

${ }^{7} \mathrm{nd}=$ not detected.
} 
biomarker(s) in the $24 \mathrm{~h}$ post dose urine. As shown in Table 3 and Figure 1, the amount of each biomarker excreted in the $24 \mathrm{~h}$ post dose urine was correlated with the amount of mycotoxin intake and the percentage of ingested mycotoxin that was excreted as biomarker in urine varied depending of the mycotoxin considered. The highest mean percentage of ingested mycotoxin excreted as biomarker in the $24 \mathrm{~h}$ post dose urine was observed for ZEA (28.4\%) followed by DON (27.9\%), $\alpha-Z O L(8.3 \%)$, FB $_{1}(2.6 \%)$, OTA (2.6\%), AFM (2.5\%) and DOM-1 (0.6\%) (Figure 1). These results fall within those obtained in studies previously conducted with pigs fed diets contaminated with a single or a combination of mycotoxins. For example, 14-15.6\% of ingested ZEA was excreted as ZEA $+\alpha-Z O L$ in pig urine collected during 8 $\mathrm{h}$ post dose; $\beta$-ZOL was not detected (Olsen et al., 1985; Zöllner et al., 2002). A lower urinary excretion of ZEA was reported by MacDougald et al. (1990); only 5.3\% of the ingested dose was excreted in the $8 \mathrm{~h}$ urine following ingestion of ZEA. The higher values of excretion observed in our study could be related to the longer period ( $24 \mathrm{~h}$ ) of urine collection.

Our results of urinary excretion of DON (27.9\%) and DOM$1(0.6 \%)$ are in agreement with those reported in other studies wherein a range of $23-60 \%$ of ingested DON or 3-ADON was eliminated as DON, whereas a range of $0-3 \%$ was excreted as DOM-1 (Eriksen et al., 2003; Dänicke et al., 2004a,b,c, 2005; Goyarts and Dänicke, 2006). Higher excretion of DON in pig $24 \mathrm{~h}$ urine (54-82\%) was reported

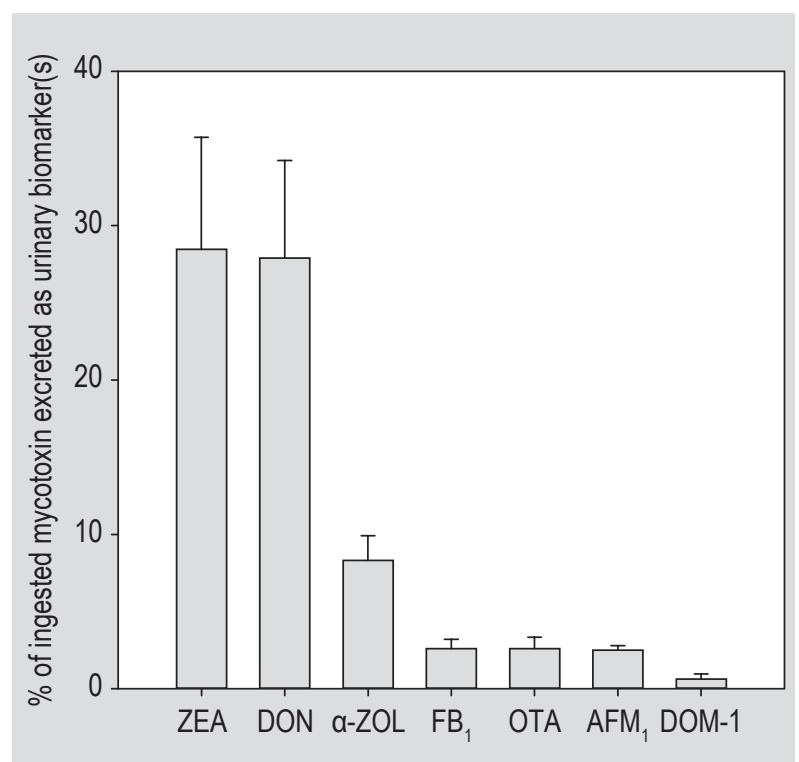

Figure 1. Mean percentage of ingested mycotoxins excreted as biomarker in $24 \mathrm{~h}$ urine. Each result is the mean \pm standard error of 16 measurements in urine from 16 piglets (values of ingested mycotoxin and excreted biomarker are given in Tables 2 and 3 , respectively). ZEA = zearalenone, $\mathrm{DON}=$ deoxynivalenol, $\alpha-Z O L$ $=\alpha$-zearalenol, $F_{1}=$ fumonisin $B_{1}$, OTA $=$ ochratoxin $A, A F M_{1}=$ aflatoxin $M_{1}$, DOM-1 = de-epoxy-deoxynivalenol. by Prelusky et al. (1988) after intragastrical administration of radioactive-labelled DON. The mean percentage of urinary excretion observed in this study for $\mathrm{FB}_{1}(2.6 \%)$ is comparable with the range of $0.7-2.5 \%$ reported in previous studies conducted with pigs using similar or different experimental conditions (Dilkin et al., 2003, 2010; Fodor et al., 2006, 2008; Szabó-Fodor et al., 2008; Prelusky et al., 1994). Few animal studies with pigs reported the urinary excretion of OTA after oral administration of the toxin. Blank and Wolffram (2004) reported that daily excretion of OTA in pig urine was 5.5\% after a single ingested dose of $2,423 \mu \mathrm{g}$ OTA equivalent to $66 \mu \mathrm{g} / \mathrm{kg}$ body weight. In our study, a lower percentage of OTA excretion was observed (2.6\%) following a lower OTA ingestion (0.16-1.32 $\mu \mathrm{g}$ / $\mathrm{kg}$ body weight). Other studies performed with pigs only reported urinary concentrations of OTA following ingestion of feed naturally contaminated with OTA. Oral exposure to diets contaminated with 38-552 and 130-790 $\mu \mathrm{g} / \mathrm{kg}$ of OTA resulted in urinary concentrations of 7.1-19.4 and $52.4 \mathrm{ng} / \mathrm{ml}$ (mean), respectively (Stoev et al. 1998, 2001). The mean percentage of ingested $\mathrm{AFB}_{1}$ excreted as urinary $\operatorname{AFM}_{1}(2.5 \%)$ found in this study was within the percentages reported in previous studies with pigs, ranging between 0.05 and $4 \%$ (Thieu, 2010).

Figure 2 shows the relationship between mycotoxin dose and urinary excretion of the relevant biomarker(s) over $24 \mathrm{~h}$ following oral administration for each mycotoxin/biomarker couple. The mycotoxin doses in the contaminated boluses and in the control diet consumed by the piglets the day before administration of the boluses were used in the graphs. The amount of each biomarker excreted in control urine collected during $24 \mathrm{~h}$ before administration of the boluses is also reported in these graphs. The lines were not forced through the origin. Linear regression analyses revealed correlation coefficients of $0.71(P=0.0001), 0.78(P<0.0001)$, $0.76(P<0.0001), 0.76(P<0.0001), 0.77(P<0.0001)$ and 0.71 $(P=0.0001)$ for the mycotoxin/biomarker couples DON/ DON, DON/DOM-1, AFB $1 / \mathrm{AFM}_{1}, \mathrm{FB}_{1} / \mathrm{FB}_{1}, \mathrm{ZEA} / \alpha-\mathrm{ZOL}$ and $\mathrm{ZEA} / \mathrm{ZEA}$, respectively. A lower correlation coefficient ( $\mathrm{r}=0.68, P=0.0002)$ was observed for OTA due to the low excretion at the highest dose. A better correlation coefficient $(r=0.95, P<0.0001)$ was obtained by eliminating the highest dose from the graph. A slight improvement was also observed for DON/DON, AFB $1 / \mathrm{AFM}_{1}, \mathrm{FB}_{1} / \mathrm{FB}_{1}$, $\mathrm{ZEA} / \alpha-\mathrm{ZOL}$ and $\mathrm{ZEA} / \mathrm{ZEA}$ by eliminating the highest dose from the graph (data not shown). These results suggest that there is a lower biomarker excretion at the highest dose, which is probably connected with a reduced and poorly reproducible absorption at the gastrointestinal level. This effect was particularly evident for OTA, although a good reproducibility of OTA excreted was observed at the highest dose (Figure 2). A better correlation was observed for DON/DOM-1 (r=0.78, $P<0.0001)$ compared to DON/ $\operatorname{DON}(\mathrm{r}=0.71, P=0.0001)$, suggesting that DOM-1 could be a better exposure biomarker for DON in pigs. However, the 
A
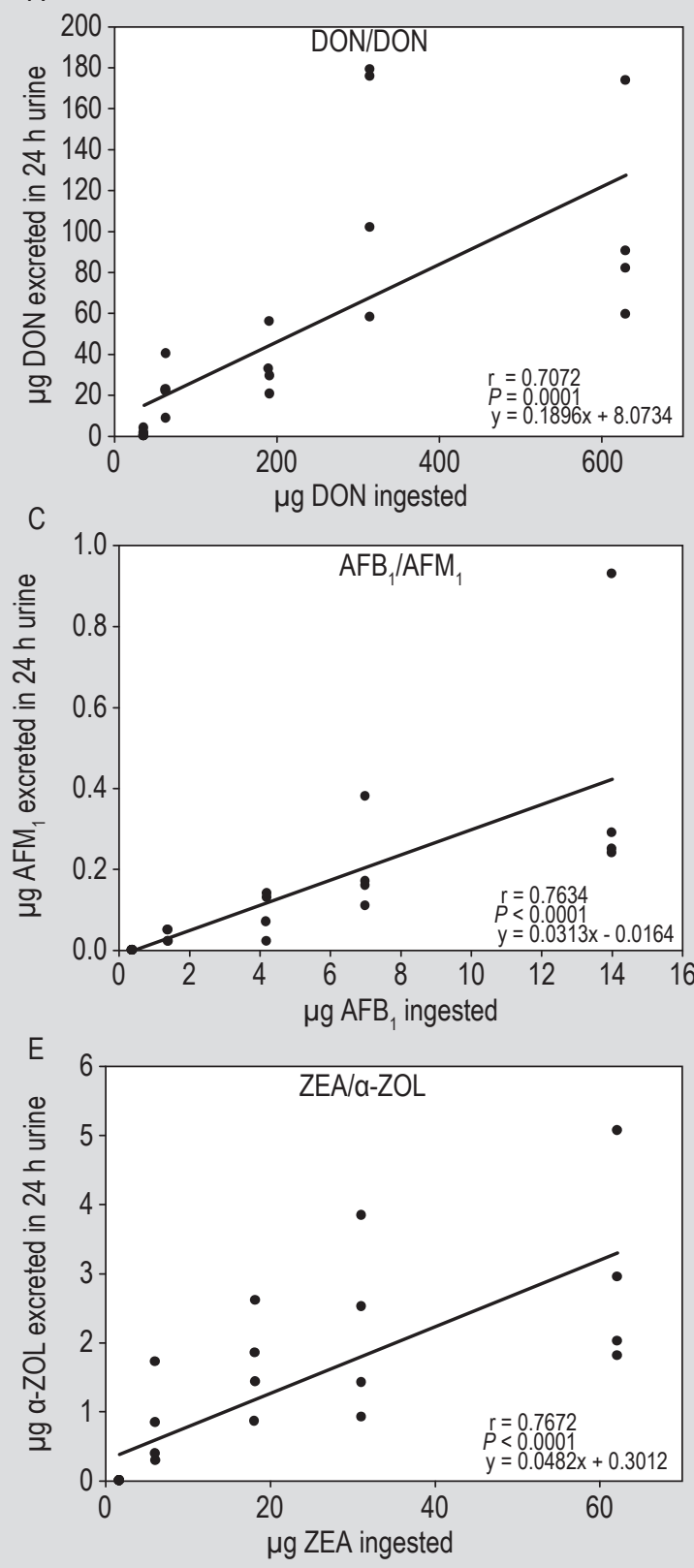

G
B

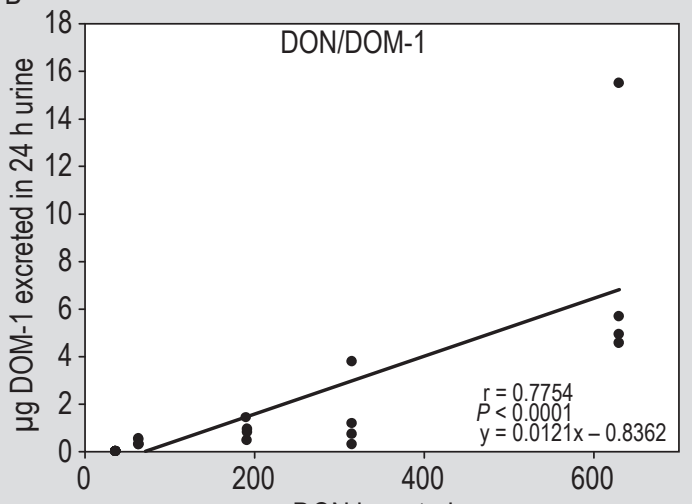

$\mu \mathrm{g}$ DON ingested
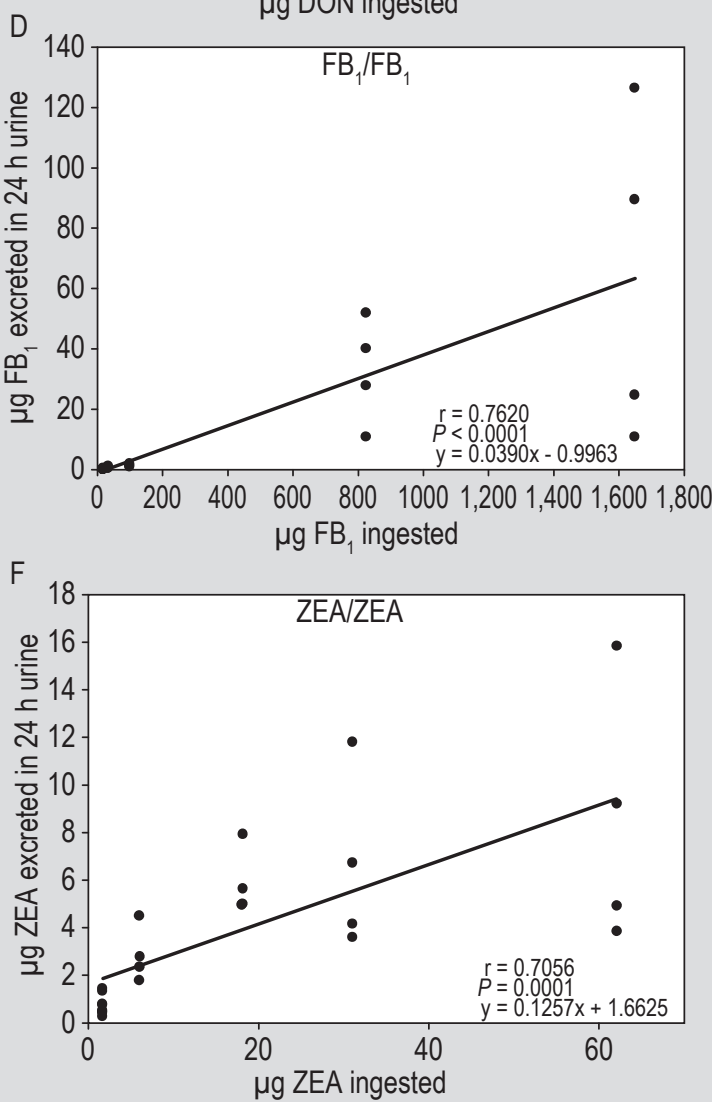

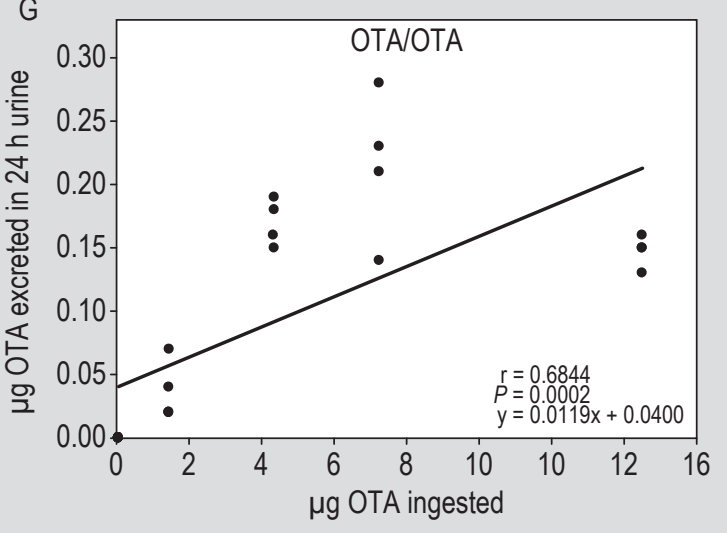

Figure 2. Linear regression analysis of ingested mycotoxin dose compared to the relevant urinary biomarker(s) excreted in $24 \mathrm{~h}$ post dose for each mycotoxin/biomarker couple. (A) Deoxynivalenol (DON) / DON, (B) DON / de-epoxy-deoxynivalenol (DOM-1),

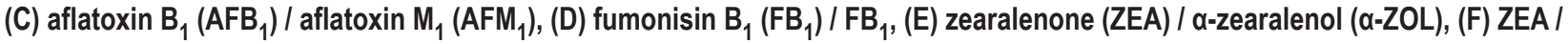
ZEA, and (G) ochratoxin A (OTA) / OTA. 
urinary concentrations of DOM-1 were on average about 30 times lower than those of DON (Table 3). These results suggest that the proposed method for DON is also robust at low concentrations using DOM-1 as urinary biomarker, although DON should be preferred as biomarker when low exposure levels need to be monitored or a less sensitive analytical method is available. The same situation was observed for ZEA/ $\alpha-Z O L(r=0.77, P<0.0001)$ and ZEA/ ZEA ( $\mathrm{r}=0.71, P=0.0001)$ and again the mean urinary concentrations of ZEA were 3 times higher than those of $\alpha$-ZOL (Table 3), which makes ZEA a more sensitive biomarker for piglets. In dose-response experiments performed by Dänicke et al. (2005) with adult gilts, mean urinary concentrations of $\alpha$-ZOL were about $30 \%$ higher than those of ZEA. The use of piglets in our experiment compared to adult gilts used by Dänicke et al. (2005) should explain this difference. Adults gilts have a mature metabolism system that should be capable to transform higher amounts of ZEA into $\alpha$-ZOL compared to piglets.

Urinary $\mathrm{AFM}_{1}, \mathrm{FB}_{1}$, OTA and $\mathrm{DON}$ are recognised as good biomarkers of exposure to $\mathrm{AFB}_{1}, \mathrm{FB}_{1}$, OTA and DON, respectively, for humans, with adjusted correlation coefficients ranging from 0.52 to 0.91 (Turner et al., 2012). The results reported in this study fall within this range including those obtained for ZEA, $\alpha-Z O L$ and DOM-1. Figure 2 also shows a difference between excreted amounts of biomarkers of individual animals receiving the same dose. For some biomarkers or dose, a high variability of results was observed. However, a high variability is common for in vivo studies and our results with piglets are comparable to or lower than those reported by other authors (Blank and Wolffram, 2004; Dänicke et al., 2005; Fodor et al., 2006; Stoev et al., 2001; Thieu and Pettersson, 2009). We cannot exclude that matrix effects may have influenced the precision of results obtained in this study. However, we are confident that the variability of the biomarker levels observed in individual animals receiving the same contaminated bolus is animal dependent according to the results reported by other authors that were obtained with methods (HPLC-UV/FD) that do not suffer from unpredictable signal suppression or enhancement (Blank and Wolffram, 2004; Dänicke et al., 2005; Thieu and Pettersson, 2009).

\section{Conclusions}

This is the first in vivo study that evaluated the doseresponse between simultaneous oral ingestion of $\mathrm{FB}_{1}$, OTA, DON, ZEA and $\mathrm{AFB}_{1}$ and urinary excretion of the relevant biomarkers within $24 \mathrm{~h}$ after dosage by using a mass balance approach. The good sensitivity of the LCMS/MS method used in this study permitted to detect and quantify low levels of most of the investigated biomarkers in control urine samples collected before administration of contaminated boluses. In particular, low levels of DON,
DOM-1, FB 1 , ZEA, $\alpha-Z O L$ and OTA were measured in $62,44,56,100,19$ and $44 \%$ of control urines, respectively. The method also permitted to detect and quantify urinary biomarkers of mycotoxins at the doses investigated. The mass balance and the multi-mycotoxin approaches allowed to assess the simultaneous excretion rate of five mycotoxins in urine of piglets as specific biomarkers. A positive dose-response relationship for the tested combinations of mycotoxin/biomarker has been demonstrated, which suggest possible ranges of mycotoxin doses for future studies. The good dose-response correlations make the urinary multi-biomarker approach a good tool to assess in vivo the efficacy of mycotoxin detoxifying agents in reducing the bioavailability of mixtures of $\mathrm{DON}, \mathrm{FB}_{1}, \mathrm{AFB}_{1}$, OTA and ZEA.

\section{Acknowledgements}

This work was supported by EU-FP7 project MYCORED (KBBE. 2007-222690).

\section{References}

Avantaggiato, G., Solfrizzo, M. and Visconti, A., 2005. Recent advances on the use of adsorbent materials for detoxification of Fusarium mycotoxins. Food Additives and Contaminants 22: 379-388.

Blank, R. and Wolffram, S., 2004. Alkalinization of urinary pH accelerates renal excretion of ochratoxin A in pigs. Journal of Nutrition 134: 2355-2358.

Boudergue, C., Burel, C., Dragacci, S., Favrot, M.C., Fremy, J.M., Massimi, C., Prigent, P., Debongnie, P., Pussemier, L., Boudra, H., Morgavi, D., Oswald, I., Perez, A. and Avantaggiato, G., 2009. Review of mycotoxin-detoxifying agents used as feed additives: mode of action, efficacy and feed/food safety. Scientific report submitted to EFSA. Available at: http://www.scribd.com/doc/58481519/EFSAReport-on-Mycotoxin-Detoxifying-Agents-2.

Council for Agricultural Science and Technology (CAST), 2003. Mycotoxins: risks in plant, animal and human systems. Task force report No. 139. CAST, Ames, IA, USA.

Dänicke, S., Brüssow, K.P., Valenta, H., Ueberschär, K.H., Tiemann, U. and Schollenberger, M., 2005. On the effects of graded levels of Fusarium toxin contaminated wheat in diets for gilts on feed intake, growth performance and metabolism of deoxynivalenol and zearalenone. Molecular Nutrition and Food Research 49: 932-943.

Dänicke, S., Goyarts, T., Valenta, H., Razzazi, E. and Böhm, J., 2004c. On the effects of deoxynivalenol (DON) in pig feed on growth performance, nutrients utilization and DON metabolism. Journal of Animal and Feed Sciences 13: 539-556.

Dänicke, S., Valenta, H., Döll, S., Ganter, M. and Flachowsky, G., 2004a. On the effectiveness of a detoxifying agent in preventing fusariotoxicosis in fattening pigs. Animal Feed Science and Technology 114: 141-157. 
Dänicke, S., Valenta, H., Klobasa, F., Döll, S., Ganter, M. and Flachowsky, G., 2004b. Effects of graded levels of Fusarium toxin contaminated wheat in diets for fattening pigs on growth performance, nutrient digestibility, deoxynivalenol balance and clinical serum characteristics. Archives of Animal Nutrition 58: 1-17.

De Andrés, F., Zougagh, M., Castañeda, G. and Ríos, A., 2008. Determination of zearalenone and its metabolites in urine samples by liquid chromatography with electrochemical detection using a carbon nanotube-modified electrode. Journal of Chromatography A 1212: 54-60.

Dilkin, P., Direito, G., Sismas, M.M.S., Mallman, C.A. and Corrêa, B., 2010. Toxicokinetics and toxicological effects of single oral dose of fumonisin $\mathrm{B}_{1}$ containing Fusarium verticillioides culture material. Chemico-Biological Interactions 185: 157-162.

Dilkin, P., Zorzete, P., Mallmann, C.A., Gomes, J.D.F., Utiyama, C.E., Oetting, L.L. and Corrêa, B., 2003. Toxicological effects of chronic low doses of aflatoxin $\mathrm{B}_{1}$ and fumonisin $\mathrm{B}_{1}$-containing Fusarium moniliforme culture material in weaned piglets. Food and Chemical Toxicology 41: 1345-1353.

Ediage, E.N., Di Mavungu, J.D., Song, S., Wu, A., Van Peteghem, C. and De Saeger, S., 2012. An LC-MS/MS method for multi-detection of mycotoxin biomarkers in human urine. Analytica Chimica Acta 741: 58-69.

Eriksen, G.S., Pettersson, H. and Lindberg, J.E., 2003. Absorption, metabolism and excretion of 3-acetyl DON in pigs. Archives of Animal Nutrition 57: 335-345.

European Commission (EC), 2003. Commission Directive 2003/100/ EC of 31 October 2003 amending Annex I to Directive 2002/32/ EC of the European Parliament and of the Council on undesirable substances in animal feed. Official Journal of the European Union L285: 33-37.

European Commission (EC), 2006. Commission Recommendation 2006/576/EC of 17 August 2006 on the presence of deoxynivalenol, zearalenone, ochratoxin A, T-2 and HT-2 and fumonisins in products intended for animal feeding. Official Journal of the European Union L229: 7-9.

European Committee for Standardisation (CEN), 2006. EN/ISO 17375. Determination of aflatoxin $\mathrm{B}_{1}$. CEN, Brussels, Belgium.

European Committee for Standardisation (CEN), 2009. EN 15791. Determination of deoxynivalenol in animal feed - HPLC method with immunoaffinity column clean-up. CEN, Brussels, Belgium.

European Committee for Standardisation (CEN), 2009. EN 15792. Determination of zearalenone in animal feed - High performance liquid chromatographic method with fluorescence detection and immunoaffinity column clean-up. CEN, Brussels, Belgium.

European Committee for Standardisation (CEN), 2012. EN 16006. Determination of the sum of fumonisin B1 \& B2 in compound animal feed with immunoaffinity clean-up and RP-HPLC with fluorescence detection after pre- or post-column derivatisation. CEN, Brussels, Belgium.

European Committee for Standardisation (CEN), 2012. EN 16007. Determination of ochratoxin A in animal feed by immunoaffinity column clean-up and high performance liquid chromatography with fluorescence detection. CEN, Brussels, Belgium.
European Food Safety Authority (EFSA), 2010. Statement on the establishment of guidelines for the assessment of additives from the functional group 'substances for reduction of the contamination of feed by mycotoxins'. EFSA Journal 8: 1693.

Fodor, J., Balogh, K., Weber, M., Méézes, M., Kametler, L., Póósa, R., Mamet, R., Bauer, L., Horn, P., Kováács, F. and Kováács, M., 2008. Absorption, distribution and elimination of fumonisin $B_{1}$ metabolites in weaned piglets. Food Additives and Contaminants Part A 25: 88-96.

Fodor, J., Meyer, K., Riedlberger, M., Bauer, J., Horn, P., Kovacs, F. and Kovacs, M., 2006. Distribution and elimination of fumonisin analogues in weaned piglets after oral administration of Fusarium verticillioides fungal culture. Food Additives and Contaminants 23: 492-501.

Goyarts, T. and Dänicke, S., 2006. Bioavailability of the Fusarium toxin deoxynivalenol (DON) from naturally contaminated wheat for the pig. Toxicology Letters 163: 171-182.

Grenier, B. and Oswald, I.P., 2011. Mycotoxin co-contamination of foods and feeds: meta-analysis of publications describing toxicological interactions. World Mycotoxin Journal 4: 285-313.

Ho, C.C., 1987. The metabolites of aflatoxins $B_{1}$ and $G_{1}$ in the pig urine. Journal of the Chinese Society of Veterinary Science 13: 197-201.

MacDougald, O., Thulin, A. and Pestka, J., 1990. Determination of zearalenone and related metabolites in porcine urine by modified enzyme-linked immunosorbent assay. Journal of AOAC International 73: 65-68.

Meissonnier, G.M., Raymond, I., Laffitte, J., Cossalter, A.M., Pinton, P., Benoit, E., Bertin G., Galtier, P. and Oswald, I.P., 2009. Dietary glucomannan improves the vaccinal response in pigs exposed to $\mathrm{AFB}_{1}$ or T-2 toxin. World Mycotoxin Journal 2: 161-172.

Olsen, M.E., Petterson, H.I. and Sandholm, K.A., 1985. Quantitative liquid chromatographic method using fluorescence detection for determining zearalenone and its metabolites in blood plasma and urine. Journal of AOAC International 68: 632-635.

Polychronaki, N., Wild, C.P., Mykkänen, H., Amra, H., Abdel-Wahhab, M., Sylla, A., Diallo, M., El-Nezami, H. and Turner, P.C., 2008. Urinary biomarkers of aflatoxin exposure in young children from Egypt and Guinea. Food and Chemical Toxicology 46: 519-526.

Prelusky, D.B., Hartin, K.E., Trenholm, H.L. and Miller, J.D., 1988. Pharmacokinetic fate of ${ }^{14} \mathrm{C}$-labelled deoxynivalenol in swine. Fundamental and Applied Toxicology 10: 276-286.

Prelusky, D.B., Trenholm, H.L. and Savard, M.E., 1994. Pharmacokinetic fate of ${ }^{14}$ C-labelled fumonisin B1 in swine. Natural Toxins 2: 73-80.

Scott, P.M, 2005. Biomarkers of human exposure to ochratoxin A. Food Additives and Contaminants 22: 99-107.

Shephard, G.S., Van der Westhuizen, L. and Sewram, V., 2007. Biomarkers of exposure to fumonisin mycotoxins: a review. Food Additives and Contaminants 24: 1196-1201.

Solfrizzo, M., Carratù, M.R., Avantaggiato, G., Galvano, F., Pietri, A. and Visconti, A., 2001b. Ineffectiveness of activated carbon in reducing the alteration of sphingolipid metabolism in rats exposed to fumonisin contaminated diets. Food and Chemical Toxicology 39: 507-511. 
Solfrizzo, M., Gambacorta, L., Lattanzio, M.T.V., Powers, S. and Visconti, A., 2011. Simultaneous LC-MS/MS determination of aflatoxin $\mathrm{M}_{1}$, ochratoxin $\mathrm{A}$, deoxynivalenol, de-epoxydeoxynivalenol, $\alpha$ - and $\beta$-zearalenols and fumonisin $B_{1}$ in urine as a multibiomarker method to assess exposure to mycotoxins. Analytical and Bioanalytical Chemistry 401: 2831-2841.

Solfrizzo, M., Visconti, A., Avantaggiato, G., Torres, A. and Chulze, S., 2001a. In vitro and in vivo studies to assess the effectiveness of cholestyramine as a binding agent for fumonisins. Mycopathologia 151: 147-153.

Stoev, S.D., Paskalev, M., MacDonald, S. and Mantle, P.G., 2002. Experimental one year ochratoxin A toxicosis in pigs. Experimental and Toxicological Pathology 53: 481-487.

Stoev, S.D., Stoeva, J.K., Anguelov, G., Hald, B., Creppy, E.E. and Radic, B., 1998. Haematological, biochemical and toxicological investigations in spontaneous cases with different frequency of porcine nephropathy in Bulgaria. Journal of Veterinary Medicine A 45: 229-236.

Stoev, S.D., Vitanov, S., Anguelov, G., Petkova-Bocharova, T. and Creppy, E.E., 2001. Experimental mycotoxic nephropathy in pigs provoked by a diet containing ochratoxin A and penicillic acid. Veterinary Research Communications 25: 205-223.

Streit, E., Schatzmayr, G., Tassis, P., Tzika, E., Marin, D., Taranu, I., Tabuc, C., Nicolau, A., Aprodu, I., Puel, O. and Oswald, I.P., 2012. Current situation of mycotoxin contamination and co-occurrence in animal feed - focus on Europe. Toxins 4: 788-809.
Szabó-Fodor, J., Kametler, L., Pósa, R., Mamet, R., Rajli, V., Bauer, J., Horn, P., Kovács, F. and Kovács, M., 2008. Kinetics of fumonisin $B_{1}$ in pigs and persistence in tissues after ingestion of a diet containing a high fumonisin concentration. Cereal Research Communications 36: 331-336.

Thieu, N.Q. and Petterson, H., 2009. Zearalenone, deoxynivalenol and aflatoxin $\mathrm{B}_{1}$ and their metabolites in urine as biomarkers for mycotoxin exposure. Mycotoxin Research 25: 59-66.

Thieu, N.Q., 2010. Evaluation of the exposure of pigs to mycotoxins by their biomarkers. In: Proceedings of the $4^{\text {th }}$ Biomin World Nutrition Forum. Biomin, Salzburg, Austria, pp. 67-72.

Turner, P.C., Flannery, B., Isitt, C., Ali, M. and Pestka, J., 2012. The role of biomarkers in evaluating human health concerns from fungal contaminants in food. Nutrition Research Reviews 25: 162-179.

Turner, P.C., White, K.L., Burley, V.J., Hopton, R.P., Rajendram, A., Fisher, J., Cade, J.E. and Wild, C.P., 2010. A comparison of deoxynivalenol intake and urinary deoxynivalenol in UK adults. Biomarkers 15: 553-562.

Warth, B., Sulyok, M., Fruhmann, P., Mikula, H., Berthiller, F., Schuhmacher, R., Hametner, C., Abia, W.A., Adam, G., Fröhlich, J. and Krska, R., 2012. Development and validation of a rapid multibiomarker liquid chromatography/tandem mass spectrometry method to assess human exposure to mycotoxins. Rapid Communications in Mass Spectrometry 26: 1533-1540.

Zöllner, P., Jodlbauer, J., Kleinova, M., Kahlbacher, H., Kuhn, T., Hochsteiner, W. and Lindner, W., 2002. Concentration levels of zearalenone and its metabolites in urine, muscle tissue, and liver samples of pigs fed with mycotoxin-contaminated oats. Journal of Agricultural and Food Chemistry 50: 2494-2501. 